\title{
Strange quark stars within the Nambu-Jona-Lasinio model
}

\author{
M. Hanauske ${ }^{1}$, L.M. Satarov ${ }^{1,2}$, I.N. Mishustin ${ }^{1,2,3}$, H. Stöcker ${ }^{1}$, and W. Greiner ${ }^{1}$ \\ ${ }^{1}$ Institut für Theoretische Physik, J. W. Goethe-Universität, D-60054 Frankfurt, Germany \\ ${ }^{2}$ The Kurchatov Institute, 123182 Moscow, Russia \\ ${ }^{3}$ The Niels Bohr Institute, DK-2100 Copenhagen O, Denmark
}

\begin{abstract}
We investigate the properties of charge-neutral $\beta$-equilibrium cold quark matter within the Nambu-Jona-Lasinio model. The calculations are carried out for different ratios of coupling constants characterizing the vector and scalar 4 -fermion interaction, $\xi \equiv G_{V} / G_{S}$. It is shown that for $\xi<0.4$ matter is self-bound and for $\xi<0.65$ it has a first order phase transition of the liquidgas type. The Gibbs conditions in the mixed phase are applied for the case of two chemical potentials associated with the baryon number and electric charge. The characteristics of the quark stars are calculated for $\xi=0,0.5$ and 1. It is found that the phase transition leads to a strong density variation at the surface of these stars. For $\xi=1$ the properties of quark stars show behaviors typical for neutron stars. At $\xi \gtrsim 0.4$ the stars near to the maximum mass have a large admixture of strange quarks in their interiors.
\end{abstract}

PACS number: 14.65.-q, 26.60.+c, 97.10.-q

\section{INTRODUCTION}

The direct application of QCD at moderate temperatures and nonzero baryon densities is not possible at present. Therefore, more simple effective models respecting some basic symmetry properties of QCD are commonly used. The Nambu-Jona-Lasinio (NJL) model 
[1:2] which is dealing with constituent quarks and respects chiral symmetry is one of the most popular model of this kind. In recent years this model has been widely used for describing hadron properties (see reviews [3,4]), phase transitions in dense matter [5] and multiparticle bound states [8 10]. This model has been also applied for studying the Equation of State $($ EoS $)$ of $\beta$-equilibrated matter appropriate for stars [11,12]. Unfortunately, the repulsive vector interaction, which is very important in dense baryon-rich environment [6.9], was not taken into account there. The main goal of our paper is to study the influence of this interaction on the EoS and the structure of quark stars. We assume that these stars are composed of pure quark matter, similar to previous studies using a simplified EoS of the MIT bag model [13,14. We do not consider hybrid stars where the quark matter is matched to the hadron matter at low densities. The reason of this is two-fold. First, all matching procedures are quite ambiguous and model dependent. Second, our calculations show that the interiors of such stars have so high densities that the quark degrees of freedom seem to be more appropriate. At least this should be a reasonable first approximation to hybrid stars. One more advantage of using quark degrees of freedom is related to the problem of strangeness content of the star matter. In recent years many calculations have been done in hadronic models where strangeness appears through either hyperon formation [15] or kaon condensation [16 18]. Within the NJL model the strangeness degree of freedom is handled in a very simple way through the population of matter by strange quarks at sufficiently high baryon densities.

\section{II. $\beta$-EQUILIBRIUM QUARK MATTER}

\section{A. Description of the model}

Below we use the SU(3)-flavour version of the NJL model proposed in Ref. [19], but including not only the scalar but also the vector 4 -fermion interaction. The color singlet part of the Lagrangian in the mean field approximation can be written as [10] $(\hbar=c=1)$ 


$$
\begin{aligned}
\mathcal{L} & =\sum_{f} \bar{\psi}_{f}\left(i \not \partial-m_{f}-\gamma_{0} G_{V} \rho_{f}\right) \psi_{f}-\frac{G_{S}}{2} \sum_{f} \rho_{S f}^{2} \\
& +\frac{G_{V}}{2} \sum_{f} \rho_{f}^{2}+4 K \prod_{f} \rho_{S f}+\mathcal{L}_{e} .
\end{aligned}
$$

Here $\psi_{f}$ is the field operator of quarks with flavour $f=u, d, s$ and

$$
\begin{aligned}
\rho_{S f} & =<\bar{\psi}_{f} \psi_{f}> \\
\rho_{f} & =<\bar{\psi}_{f} \gamma_{0} \psi_{f}>
\end{aligned}
$$

are their scalar and vector densities. Angular brackets in Eqs. (2)-(3) denote quantumstatistical averaging. $G_{S}, G_{V}$ and $K$ in Eq. (I) are respectively the coupling constants of scalar, vector and flavour-mixing interactions.

The last term in Eq. (11) is the leptonic part of the Lagrangian. Below we take into account only electrons and treat them as ideal gas of massless particles. As calculation show, the maximum value of the electron chemical potential $\mu_{e}$ does not exceed significantly the muon mass so that the muon admixture should be small. In this approximation

$$
\mathcal{L}_{e}=\bar{\psi}_{e} i \not \partial \psi_{e}
$$

where $\psi_{e}$ is the electron field.

The constituent quark masses, $m_{f}$, are determined from the coupled set of gap equations

$$
m_{f}=m_{0 f}-G_{S} \rho_{S f}+2 K \prod_{f^{\prime} \neq f} \rho_{S f^{\prime}}
$$

where $m_{0 f}$ is the bare (current) mass of quarks with flavour $f$. The single-particle energies of quarks are equal to $E_{f}(p)=\sqrt{m_{f}^{2}+p^{2}}$.

Within this model chiral condensates $<\bar{\psi}_{f} \psi_{f}>$ are given by the scalar densities of quarks occupying both the negative and positive energy states. The divergent contributions of negative energy states of the Dirac sea are regularized by introducing a 3-momentum cut-off $\Theta(\Lambda-p)$ in momentum space integrals. In the case of spatially homogeneous matter at zero temperature one arrives at the following expression [9] 


$$
\rho_{S f}=-\frac{\nu m_{f}}{2 \pi^{2}} \int_{p_{F f}}^{\Lambda} \frac{\mathrm{d} p p^{2}}{E_{f}(p)}=\frac{\nu m_{f}}{4 \pi^{2}}\left[p_{F f}^{2} \Phi\left(\frac{m_{f}}{p_{F f}}\right)-\Lambda^{2} \Phi\left(\frac{m_{f}}{\Lambda}\right)\right]
$$

Here $\nu=6$ is the spin-color degeneracy factor, $p_{F f}=\left(\frac{6 \pi^{2}}{\nu} \rho_{f}\right)^{1 / 3}$ is the Fermi momentum of quarks with flavour $f$ and

$$
\Phi(x)=\sqrt{1+x^{2}}-\frac{x^{2}}{2} \ln \frac{\sqrt{1+x^{2}}+1}{\sqrt{1+x^{2}}-1} .
$$

The model parameters $m_{0 f}, G_{S}, K, \Lambda$ can be fixed by reproducing the observed masses of $\pi, K$, and $\eta^{\prime}$ mesons as well as the pion decay constant $f_{\pi}$. As shown in Ref. 19, a reasonable fit is achieved with the following values:

$$
\begin{gathered}
m_{0 u}=m_{0 d}=5.5 \mathrm{MeV}, \quad m_{0 s}=140.7 \mathrm{MeV} \\
G_{S}=20.23 \mathrm{GeV}^{-2}, \quad K=155.9 \mathrm{GeV}^{-5}, \quad \Lambda=0.6023 \mathrm{GeV} .
\end{gathered}
$$

Several attempts have been made to extract the vector coupling constant by fitting the nucleon axial charge $g_{A}$ [3] and masses of vector mesons [20]. It was established that the ratio of the vector and scalar coupling constants $\xi \equiv G_{V} / G_{S}$ should be of the order of unity. But still there is no agreement between the values used by different authors. As demonstrated in Refs. [9, 10], the EoS of baryon-rich matter, in particular, the existence of bound states and phase transitions is highly sensitive to $\xi$. Due to uncertainty in the parameter $G_{V}$, below we present the results for various values of $\xi$ within the interval $0 \leq \xi \leq 1$.

\section{B. Equation of state}

We assume that the quark matter is in chemical equilibrium with respect to the strong and weak interactions. If neutrinos accompanying weak processes escape freely $\left(\mu_{\nu}=0\right)$, one obtains the following conditions

$$
\mu_{d}=\mu_{s}=\mu_{u}+\mu_{e},
$$


relating the chemical potentials $\mu_{i}$ of various particle species $i=u, d, s, e$. These equations are automatically satisfied if $\mu_{i}$ are represented as linear combinations of the baryon $\left(\mu_{B}\right)$, strange $\left(\mu_{S}\right)$ and charge $\left(\mu_{Q}\right)$ chemical potentials:

$$
\mu_{i}=B_{i} \mu_{B}+S_{i} \mu_{S}+Q_{i} \mu_{Q},
$$

where $B_{i}, S_{i}$ and $Q_{i}$ are the baryon, strangeness and charge quantum numbers of the particle species $i$. From Eqs. (10) it is evident that $\mu_{S}=0$ and $\mu_{Q}=-\mu_{e}$. In the considered case of zero temperature the chemical potentials can be written in the explicit form:

$$
\begin{aligned}
& \mu_{f}=\sqrt{m_{f}^{2}+p_{F f}^{2}}+G_{V} \rho_{f} \\
& \mu_{e} \simeq p_{F e}=\left(3 \pi^{2} \rho_{e}\right)^{1 / 3}
\end{aligned}
$$

where $\rho_{e}$ is the number density of electrons.

The chemical potentials, constituent quark masses and particle densities $\rho_{i}$ are found by simultaneously solving Eqs. (5)-(6), (10)-(13) under conditions of fixed baryon and charge densities,

$$
\begin{aligned}
& \rho_{B}=\sum_{i} B_{i} \rho_{i}=\frac{1}{3}\left(\rho_{u}+\rho_{d}+\rho_{s}\right), \\
& \rho_{Q}=\sum_{i} Q_{i} \rho_{i}=\frac{1}{3}\left(2 \rho_{u}-\rho_{d}-\rho_{s}\right)-\rho_{e} .
\end{aligned}
$$

Unless stated otherwise, the condition of local charge neutrality $\rho_{Q}=0$ is assumed.

To obtain the EoS of quark matter, one can calculate the energy density $\epsilon=T^{00}$ directly from the Lagrangian (11). At zero temperature one arrives at the expression (see Ref. [10] for details)

$$
\begin{aligned}
\epsilon & =\sum_{f}\left[\frac{\nu}{2 \pi^{2}} \int_{p_{F f}}^{\Lambda} \mathrm{d} p p^{2} E_{f}(p)+\frac{G_{S}}{2} \rho_{S f}^{2}+\frac{G_{V}}{2} \rho_{f}^{2}\right] \\
& -4 K \prod_{f} \rho_{S f}+\frac{p_{F e}^{4}}{4 \pi^{2}}-\epsilon_{\mathrm{vac}} .
\end{aligned}
$$

The constant $\epsilon_{\mathrm{vac}}$ is introduced in order to set the energy density of the physical vacuum $\left(p_{F i}=0\right)$ equal to zero. This constant can be expressed through the vacuum values of 
constituent quark masses. The latter are obtained by solving the gap equations (5) for the case $p_{F f}=0$. At zero temperature the pressure can be obtained from the energy density by using the thermodynamic identity

$$
P=\sum_{i} \mu_{i} \rho_{i}-\epsilon
$$

To characterize the flavour composition of quark matter, we introduce the strangeness fraction parameter

$$
r_{s}=\frac{|S|}{3 B}=\frac{\rho_{s}}{\rho_{u}+\rho_{d}+\rho_{s}}
$$

where $B$ and $S$ are the baryon charge and strangeness of matter.

\section{Possibility of a phase transition}

Some results concerning properties of the $\beta$-equilibrated quark matter at zero temperature are presented in Figs. 1 and 2. Fig. I shows pressure as a function of baryon density for three values of the parameter $\xi$, namely for $\xi=0,0.5$ and 1 . Below we compare our results with the predictions of the Hadron Chiral Model (HCM) [21,22] for the $\beta$-equilibrated hadronic matter. Pressure calculated in that model is also shown in Fig. 1. Fig. 2 represents equilibrium concentrations of different quark flavours in $\beta$-equilibrium matter. Comparison of the NJL model predictions for different $\xi$ reveals a strong sensitivity to this parameter . At $\xi=0$ one can see two zero-pressure points at nonzero baryon densities. They correspond to a local maximum and a local minimum in the energy per baryon $\epsilon / \rho_{B}$. In the considered case the local minimum corresponds to a bound state of quark matter. This means that finite droplets of such matter can exist in mechanical equilibrium with vacuum even without gravitational force.

At $\xi \sim 0.5$ the pressure curve $P=P\left(\rho_{B}\right)$ does not cross zero, but still contains parts unstable with respect to the baryon and charge density fluctuations. This implies the possibility of a first order phase transition of the liquid-gas type. In the case of isospin-symmetric 
matter similar phase transitions have been studied in Ref. [10,23] . As demonstrated in Fig. 3 parameters of the phase transition are rather sensitive to $\xi$. Dashed lines in this figure show the states unstable with respect to the decomposition of matter into two $(k=1,2)$ coexisting phases with different baryon $\left(\rho_{B}^{(k)}\right)$ and charge $\left(\rho_{\mathrm{Q}}^{(k)}\right)$ densities. The spatially averaged baryon density is defined as

$$
\rho_{B}=\lambda \rho_{B}^{(1)}+(1-\lambda) \rho_{B}^{(2)}
$$

where $\lambda$ is the volume fraction occupied by the denser phase $(0 \leq \lambda \leq 1)$.

The Gibbs conditions of two-phase equilibrium, relating pressures $P^{(k)}=P\left(\rho_{B}^{(k)}, \rho_{Q}^{(k)}\right)$, and chemical potentials of the two phases can be written as

$$
\begin{aligned}
& P^{(1)}=P^{(2)}, \\
& \mu_{B}^{(1)}=\mu_{B}^{(2)}, \\
& \mu_{Q}^{(1)}=\mu_{Q}^{(2)} .
\end{aligned}
$$

In accordance with the general discussion of Ref. [24], these conditions can be fulfilled simultaneously only at nonzero $\rho_{Q}^{(k)}$. As a consequence, only the "global" charge neutrality condition

$$
\lambda \rho_{Q}^{(1)}+(1-\lambda) \rho_{Q}^{(2)}=0
$$

should be satisfied in the mixed phase region. By using Eqs. (19)-(23) one can calculate the equilibrium pressure in this region as a function of baryon density $\rho_{B}$. As shown in Fig. 3 this pressure increases nearly linearly with $\rho_{B}$. Deviations from the Maxwell construction $(P=$ const $)$ are more visible at larger $\xi$. However, the mixed phase region becomes more narrow in this case and disappears completely at $\xi \simeq 0.65$. At $\xi=1$ no traces of this phase transition are present any more and the resulting EoS is rather stiff.

\section{PROPERTIES OF QUARK STARS}

In this section the above derived EoS is used to construct the models of compact stars composed of pure quark matter. As will be shown below, the properties of such stars differ 
significantly depending on whether the matter is self-bound or not. In the case of selfbound electrically-neutral matter one would expect the existence of macroscopic objects of any size above a certain critical mass determined by the surface effects. But certainly the gravitational interaction becomes more and more important when the mass of these objects grows.

The gravitational field is described in a standard way by using a spacetime-dependent metric $g_{\mu \nu}\left(x^{\alpha}\right)$ obeying the Einstein's equations [25]. We consider only spherically symmetric configurations and neglect all dynamical effects, like oscillation and rotation. Moreover, we restrict our considerations to stars without magnetic field and at zero temperature. It is also assumed that matter can be treated as an ideal fluid. Under these assumptions the Einstein's equations are reduced to the Tolman-Oppenheimer-Volkoff (TOV) equations [26]. For a given EoS and a fixed central baryon density $\rho_{c}$ the inside solution of the metric $g_{\mu \nu}(r)$, the energy density and pressure profiles can be determined by solving the TOV equations until the radius $R$ where the pressure vanishes. The outside solution is given by the Schwarzschild metric and depends only on the total gravitational mass $M$ of the star.

The low-density $\left(\epsilon<\epsilon_{\text {drip }}=4.3 \cdot 10^{11} \mathrm{~g} / \mathrm{cm}^{3}\right)$ outer layer of a star ('crust') contains mainly nuclei and electrons. To describe this nuclear crust we use the EoS suggested in Ref. [27].

\section{A. Mass-density curves}

Fig. Ð 4 represents the gravitational mass of stars as a function of central baryon density $\rho_{c}$. One can see that the maximum masses predicted by the NJL model depend strongly on the relative strength of the scalar and vector interactions $\xi$. At $\xi=0$ the maximum mass has a quite low value $M_{\max }=1.23 M_{\odot}$ with a high central baryon density $\rho_{c}^{\max }=9.2 \rho_{0}$, which is typical for quark matter stars [13,28]. For higher values of $\xi$ the EoS becomes stiffer (see Fig. 1) which results in increasing maximum mass. On the other hand, the central density $\rho_{c}^{\max }$ of the maximum-mass stars decreases with $\xi$. For $\xi=1$ the maximum-mass star has 
$M_{\max }=1.60 M_{\odot}$ and $\rho_{c}^{\max }=7.8 \rho_{0}$.

In Fig. 4 our results are compared with the predictions for neutron stars obtained within the HCM 円. The upper curve corresponds to the HCM calculation where hyperons are neglected whereas the lower one shows the results with the inclusion of hyperons. One can see that at higher $\xi$ the NJL results for quark stars become close to the predictions of the HCM (with inclusion of hyperons) for neutron stars.

\section{B. Mass-radius relations}

The calculated mass-radius relations are presented in Fig. 5. Again the predictions of the NJL model are compared with the results of the HCM. One can see that at $\xi=1$ the value of the minimal radius $R_{\min }=R\left(M_{\max }\right) \simeq 11.2 \mathrm{~km}$ predicted by the NJL model is close to the HCM prediction. Here, again we conclude that at large $\xi$ the properties of quark and neutron stars are similar.

At decreasing $\xi$ the minimal radius becomes closer to the predictions for quark stars made in Refs. [13],28]. For self-bound matter $(\xi<0.4)$ the mass-radius relation changes in a qualitative way. In this case the corresponding curves start from the origin and $M \propto R^{3}$ at small $R$ (without crust). It is not clear to us whether it is necessary to include the crust for these self-bound stars or not. But if we do this following the standard prescriptions 13. we see that the low-mass stars acquire an extended mantel of crust, which may reach hundreds of kilometers. On the other hand for quark stars with high masses (near to the maximum mass) the inclusion of the crust leads to a relatively small increase of the radii, of about several hundred meters.

\footnotetext{
${ }^{1}$ The slight differences between the results presented here and in 21] are due to improved numerics.
} 


\section{Density profiles}

Fig. 6 represents the baryon density profiles $\rho_{B}(r)$ predicted by the NJL model as well as by the HCM (with hyperons). In all cases the results are shown for the respective maximummass stars. The profile of a self-bound quark star at $\xi=0$ has a step-like behavior at the star's surface at radius $r \simeq 8 \mathrm{~km}$. Here the density jumps from $\rho_{B} \simeq 2.6 \rho_{0}$ to a much lower value corresponding to the crust density, or to zero if the crust is neglected. For $\xi=0.5$ the corresponding EoS has a phase transition at a finite (nonzero) pressure (see Sect. [IC). One can see that in this case the density in the mixed-phase region changes quite steeply but continuously. For $\xi=1$ no phase transition occurs and the baryon density decreases much smother resulting in a bigger radius. The density profile of a maximum-mass star predicted by the HCM starts at a much lower central baryon density, $\rho_{c} \simeq 6.5 \rho_{0}$, but extends to a larger radius. Comparing these results we conclude again that with increasing $\xi$ the quark stars become more and more similar to neutron stars.

\section{Strangeness content}

In this section we discuss the strangeness content of the quark matter as predicted by the NJL model. In Fig. 7 the strangeness fraction $r_{s}$ (see Eq. (18)) is shown as a function of the baryon density. One can see that the threshold density for the appearance of strange quarks

depends significantly on the parameter $\xi$ and diminishes with it. At $\rho_{B} \gtrsim 6 \rho_{0}$ the effect of the vector coupling on $r_{s}$ becomes less important. For all $\xi$ the strangeness fraction tends to $1 / 3$ that simply means that dense quark matter is composed of equal numbers of up, down and strange quarks. This is clear since the difference in bare masses of light and strange quarks becomes unimportant at high densities and the SU(3)-flavour symmetry is effectively restored. Similar to neutron stars, stable quark stars should have smaller central densities than the one corresponding to the maximum-mass star. This stability condition gives a maximum value for the strangeness fraction $r_{s}^{\max }$ in the star's center, indicated by open dots 
in Fig. 7. These values differ slightly for different $\xi$ values. Indeed, $r_{s}^{\max }$ increases from 0.25 to 0.29 when $\xi$ changes from 0 to 1 . In neutron star models dealing with charge-neutral hadronic matter the strangeness fraction due to formation of hyperons [15,21] is typically

smaller than in the quark stars. As one can see in Fig. 7, a much smaller value $r_{s}^{\max } \simeq 0.12$, is predicted by the HCM.

It is instructive to calculate the total strangeness content of a star. It is obtained by integrating the strange number density $\rho_{s}$ over the star's volume

$$
N_{s}=\int \sqrt{-g} \rho_{s} u^{0} d V=4 \pi \int_{0}^{R} r^{2} \frac{\rho_{s}}{\sqrt{1-2 m(r) / r}} d r
$$

where $u^{0}$ is the time component of the 4 -velocity of matter and $m(r)$ is the gravitational mass at the radius $r$. The ratio of the total strangeness number $N_{s}$ to the total baryon number $N_{B}$ is shown in Fig. 8 for different $\xi$ values. Due to the low central density the low-mass stars $\left(M \lesssim M_{\odot}\right)$ have a negligible strangeness content for all $\xi$. For larger masses $N_{s} / N_{B}$ is strongly sensitive to $\xi$. For the maximum-mass stars the value of $N_{s} / N_{B}$ changes from 0.07 to 0.39 when $\xi$ increases from 0 to 1 . This difference originates from the different density dependence of the strangeness fraction $r_{s}$ (see Fig. 7) in combination with the baryon density profiles of the maximum-mass stars. The stars with higher $\xi$ contain more strange particles due to a lower threshold in density. The quark stars with large strangeness content, say $N_{s} / N_{B}>0.3$ can be named strange quark stars.

\section{CONCLUSIONS}

In this paper the NJL model is used to construct the EoS of cold $\beta$-equilibrium quark matter and to study the structure of compact stellar objects. It was found that the EoS depends strongly on the relative strength of the vector and scalar interaction $\xi=G_{V} / G_{S}$. When repulsive vector interaction is small $(\xi<0.4)$ the matter is self-bound, i.e. there is a zero pressure point at a finite baryon density. At higher values of $\xi(0.4<\xi<0.65)$ the EoS has no zero-pressure points but still has a first order phase transition at non- 
vanishing pressure. The properties of the mixed phase were found by using the Gibbs conditions for the case of two independent chemical potentials [24] and assuming global charge neutrality. We have calculated the mass-density and mass-radius curves, the density profiles and strangeness distribution in such stars. It is found that maximum mass grows from $1.23 M_{\odot}$ to $1.6 M_{\odot}$ when $\xi$ increases from 0 to 1 . In the case of self-bound matter the baryon density at the star's surface varies very rapidly from a high value, corresponding to the zero-pressure point in the EoS, to nearly zero density. The radii of such stars could be quite small even if the crust is added (the minimum radius in this case is about $7 \mathrm{~km}$ ). At $\xi \sim 1$ the quark star global properties (maximum mass $\simeq 1.6 M_{\odot}$, radius $\simeq 11 \mathrm{~km}$ ) are similar to those for neutron stars. However, the quark stars have much higher strangeness content as compared with stars composed of hadronic matter with hyperons. These quark stars near their maximum masses can be aptly dubbed strange quark stars.

In the future we are planing to construct a more realistic EoS when hadronic degrees of freedom will be included at low densities.

\section{ACKNOWLEDGMENTS}

The authors are thankful to J. Schaffner-Bielich for helpful discussions. This work has been supported by the RFBR Grant No. 00-15-96590, the Alexander von Humboldt Foundation, the Graduiertenkolleg "Experimentelle und Theoretische Schwerionenphysik", GSI, BMBF, DFG, and the Hessische Landesgraduiertenförderung. 


\section{REFERENCES}

[1] Y. Nambu and G. Jona-Lasinio, Phys. Rev. 122, 345 (1961); 124, 246 (1961).

[2] V.G. Vaks and A.I. Larkin, Sov. J. JETP 13, 192 (1961).

[3] U. Vogl and W. Weise, Prog. Part. Nucl. Phys. 27, 195 (1991).

[4] S.P. Klevansky, Rev. Mod. Phys. 64, 649 (1992).

[5] M. Asakawa and Y. Yazaki, Nucl. Phys. A504, 668 (1989).

[6] S. Klimt, M. Lutz, and W. Weise, Phys. Lett. B249, 386 (1990).

[7] T.M. Schwarz, S.P. Klevansky, and G. Rapp, Phys. Rev. C 60, 055205 (1999).

[8] M. Buballa and M. Oertel, Nucl. Phys. A642, 39 (1998); Phys. Lett. B457, 261 (1999).

[9] I.N. Mishustin, L.M. Satarov, H. Stöcker, and W. Greiner, Phys. Rev. C 59, 3343 (1999).

[10] I.N. Mishustin, L.M. Satarov, H. Stöcker, and W. Greiner, Phys. Rev. C 62, 034901 $(2000)$.

[11] K. Schertler, S. Leupold, and J. Schaffner-Bielich, Phys. Rev. C 60, 025801 (1999).

[12] A.W. Steiner, M. Prakash, and J.M. Lattimer, Phys. Lett. B486, 239 (2000).

[13] F. Weber, Pulsars as Astrophysical Laboratories for Nuclear and Particle Physics (IoP, Bristol, 1999).

[14] A. Peschier, B. Kämpfer, G. Soff, Phys. Rev. C 61, 045203 (2000).

[15] J. Schaffner and I.N. Mishustin, Phys. Rev. C 53, 1416 (1996).

[16] G.E. Brown, C.H. Lee, M. Rho, and V. Thorsson, Nucl. Phys. A567, 937 (1994).

[17] J.A. Pons, S. Reddy, P.J. Ellis, M. Prakash, and J.M. Lattimer, Phys. Rev. C 62, 035803 $(2000)$.

[18] A. Ramos, J. Schaffner-Bielich and J. Wambach, nucl-th/0011003 
[19] P. Rehberg, S.P. Klevansky, and J. Hüfner, Phys. Rev. C 53, 410 (1996).

[20] A. Polleri, R.A. Broglia, P.M. Pizzochero, and N.N. Scoccola, Z. Phys. A357, 325 (1997).

[21] M. Hanauske, D. Zschiesche, S. Pal, S. Schramm, H. Stöcker, and W. Greiner, Astrophys. J. 537, 958 (2000).

[22] P. Papazoglou, D. Zschiesche, S. Schramm, J. Schaffner-Bielich, H. Stöcker, and W. Greiner, Phys. Rev. C 59, 411 (1999).

[23] I.N. Mishustin, L.M. Satarov, H. Stöcker, and W. Greiner, to be published in Phys. At. Nucl., hep-ph/0010223.

[24] N.K. Glendenning, Phys. Rev. D 46, 1274 (1992).

[25] C.W. Misner, K.S. Thorne and J.A. Wheeler, Gravitation (W.H. Freeman and Company, San Francisco, 1973).

[26] R. Tolman, Phys. Rev. 55, 364 (1939);

J.R. Oppenheimer and G.M. Volkoff, Phys. Rev. 55 (1939) 374.

[27] G. Baym, C. Pethick, and P. Sutherland, Astrophys. J. 170, 299 (1971).

[28] N.K. Glendenning, Compact Stars (Springer, New York, 1997). 


\section{FIGURES}

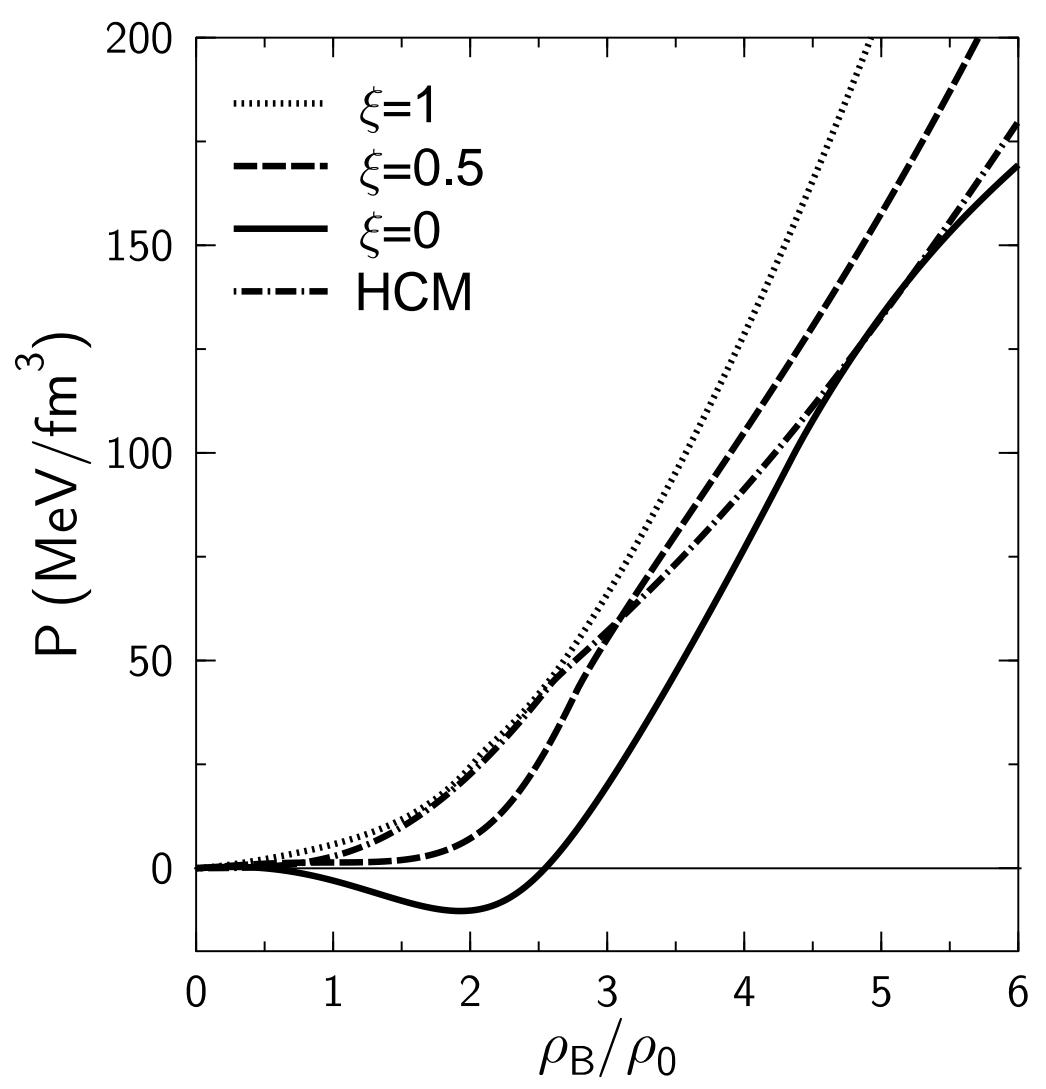

FIG. 1. Pressure as a function of the baryon density (in units of $\rho_{0}=0.15 \mathrm{fm}^{-3}$ ) calculated within the NJL model for different ratios of vector and scalar coupling constants $\xi$. In the case of $\xi=0$ the matter is self-bound that is signaled by the presence of negative pressures. Dashed-dotted line shows the predictions of the HCM (with hyperons). 


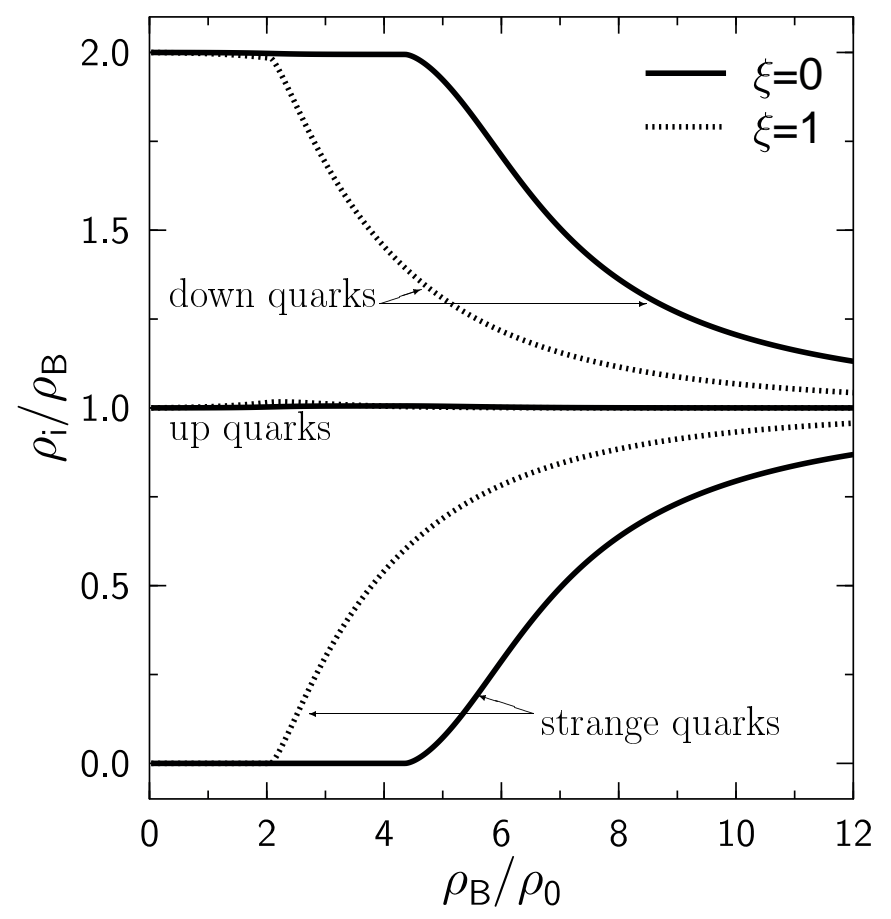

FIG. 2. Quark flavour abundances $\rho_{f} / \rho_{B}(\mathrm{f}=\mathrm{u}, \mathrm{d}, \mathrm{s})$ versus the baryon density $\rho_{B}$ for two different ratios of the vector and scalar coupling constants $\xi=0$ and 1 . 


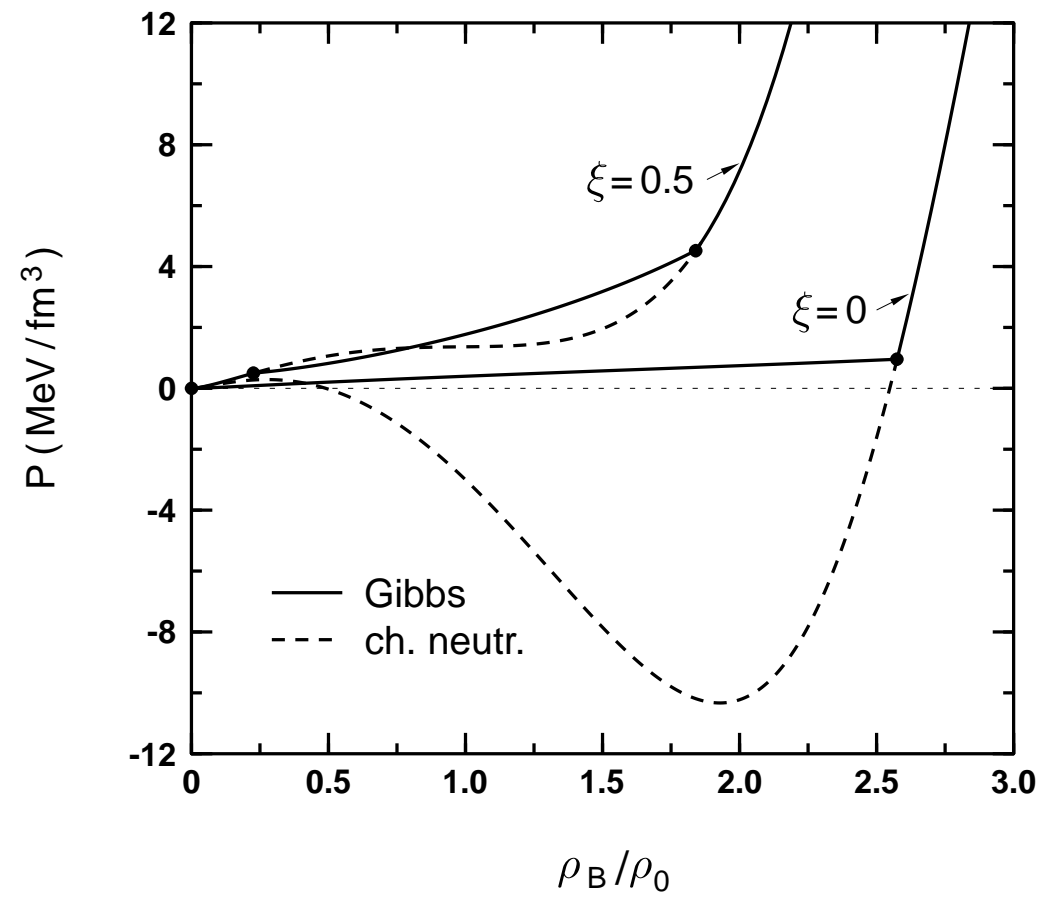

FIG. 3. Pressure as a function of the baryon density for $\xi=0$ and 0.5 when a phase transition of the liquid-gas type is predicted by the NJL model. The solid lines between the dots correspond to the mixed phase obtained by applying the Gibbs conditions. Dashed lines show the results of calculations without the mixed phase. 


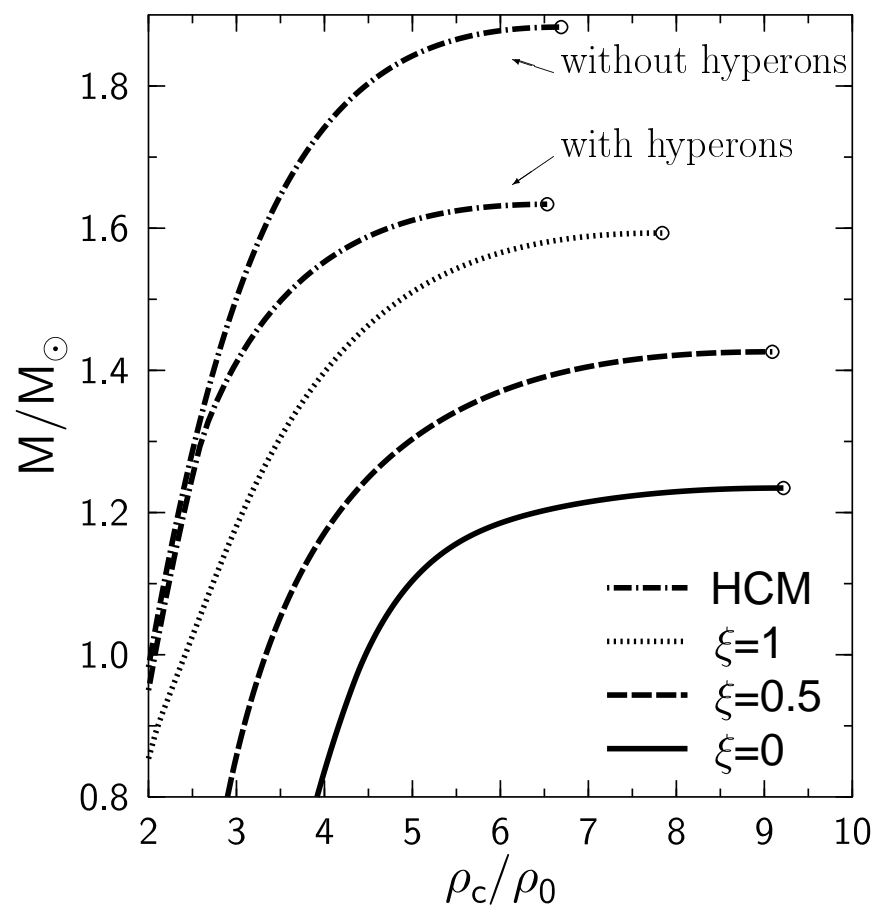

FIG. 4. Gravitational mass of a star $\mathrm{M}$ in units of the solar mass $M_{\odot}$ versus the central baryon density $\rho_{c}$. The three lower curves show the predictions for quark stars within the NJL model for different values of parameter $\xi$. The dashed-dotted curves correspond to predictions of the HCM with and without hyperons. 


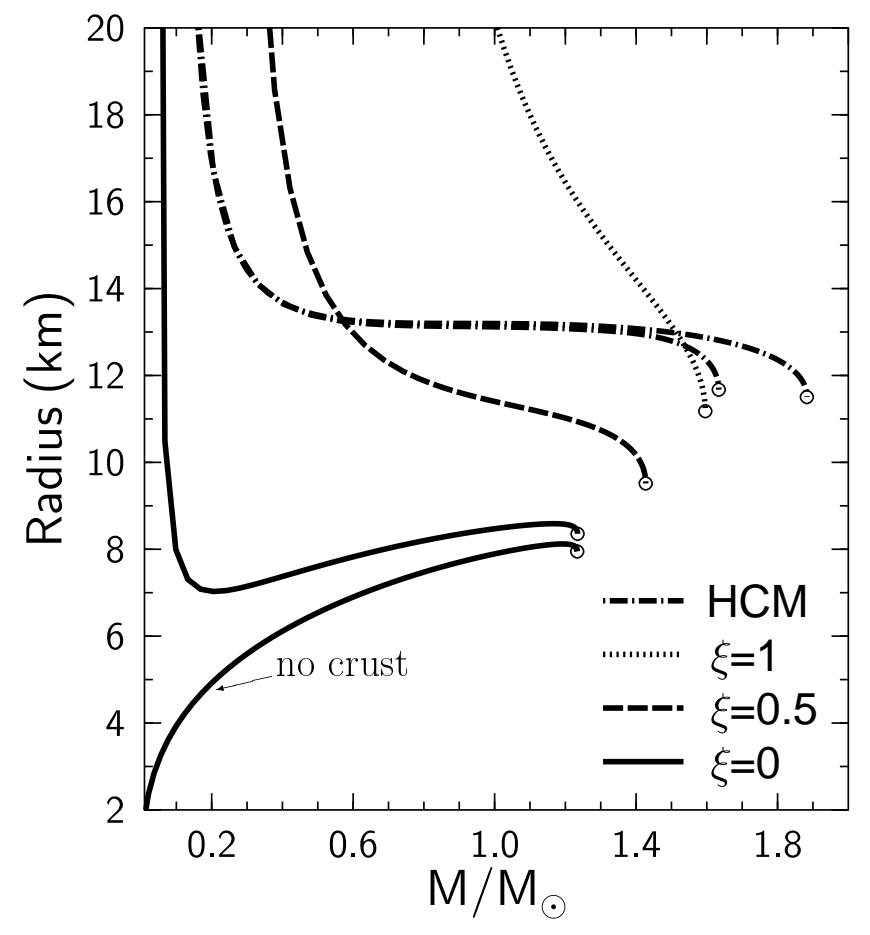

FIG. 5. Mass-radius curves predicted by the NJL model for three values of the parameter $\xi$. For comparison, the predictions of the HCM with and without hyperons are also shown. The results for self-bound stars (solid lines) are presented for two calculations with and without crust. 


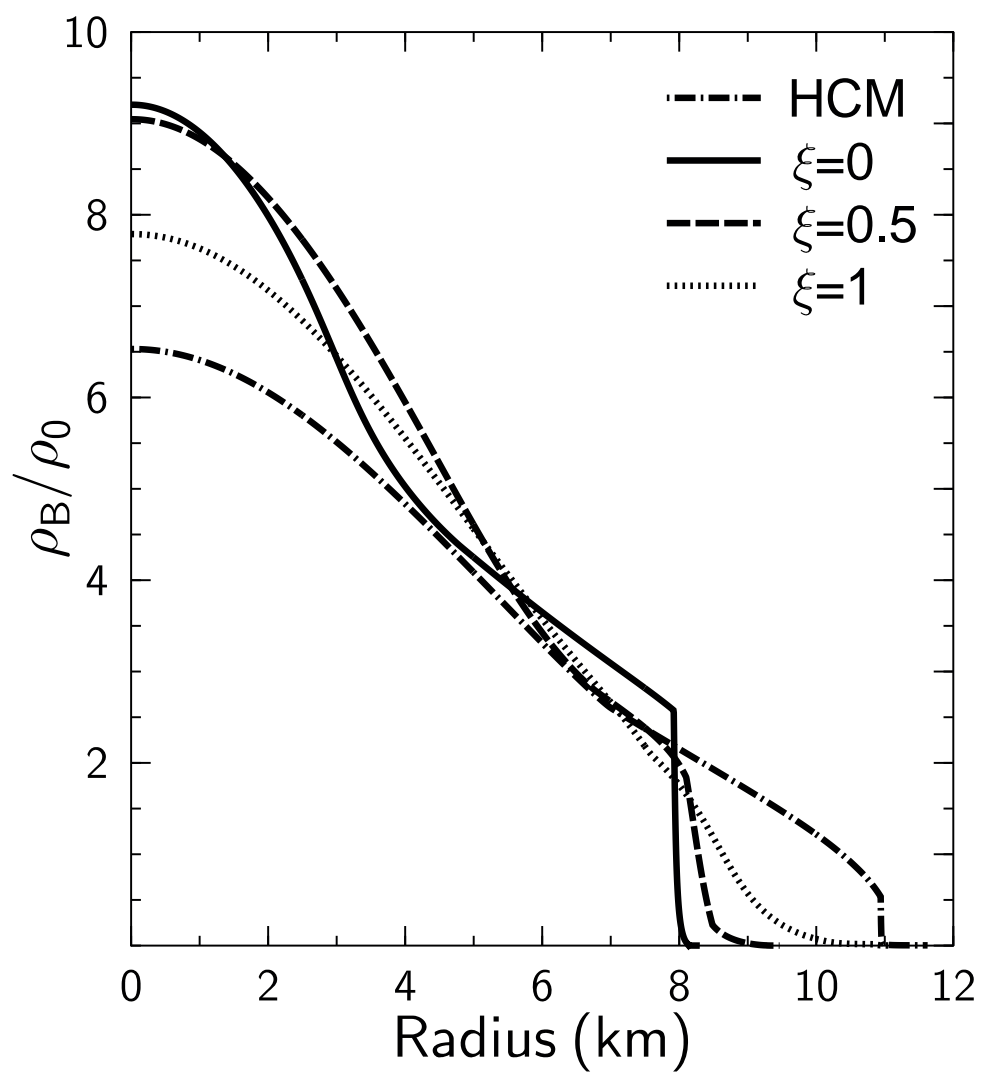

FIG. 6. Baryon density profiles for the maximum-mass quark stars predicted by the NJL model for three different values of the parameter $\xi$. The dashed-dotted line corresponds to the HCM (with hyperons). 




FIG. 7. Strangeness fraction $r_{s}$ versus the baryon density $\rho_{B}$ predicted by the NJL model for three different values of the parameter $\xi$. The dashed-dotted line shows the results of the HCM (with hyperons). The circles correspond to the values of $r_{s}$ at the center of the stars with maximum masses. 


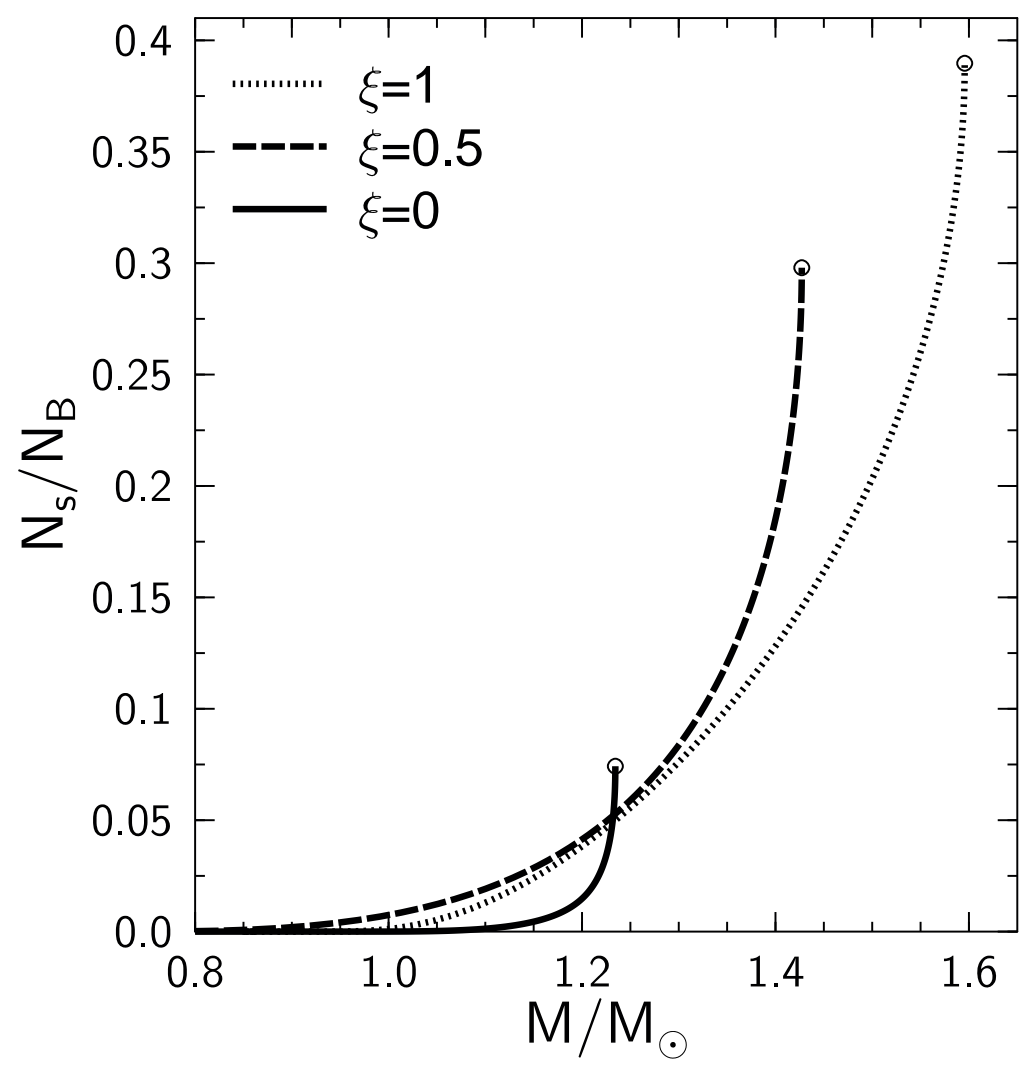

FIG. 8. The ratio of total number of strange quarks $N_{s}$ to the total baryon number $N_{B}$ as function of the gravitational mass $M$ of the quark stars predicted by the NJL model for three values of the parameter $\xi$. 